\title{
Colour transformed clustering-based water body extraction using IRS-1C LISS III image
}

\author{
Rubina Parveen* \\ Research Resource Centre, \\ Visvesvaraya Technological University, \\ Belagavi, India \\ Email: Rubina16det@gmail.com \\ *Corresponding author
}

\section{Subhash Kulkarni}

P.E.S. Institute of Technology,

Bengaluru (South Campus), India

Email: sskul@pes.edu

\section{V.D. Mytri}

APPA Institute of Engineering and Technology, Sharan Nagar, Kalaburagi, Karnataka, India

Email: appaiet@rediffmail.com

\begin{abstract}
The algorithm presented in this research article extracts and delineates the water areas using IRS-1C LISS III images. Methods available in the literature are biased with user-defined thresholds. The objective of the proposed algorithm is to provide accurate information about surface water. Initially, the input image is subjected to colour transformation clustering to extract all the similar hydrological characteristics geo-spatial features in the picture. Every cluster is then submitted to surface water detection by considering spectral information. Finally, the surface water bodies are outlined with sharp inter-regional boundaries and made visually vibrant. Thus the task of identification of water bodies is made simple, accurate and easy for the user with satisfactory qualitative analysis. Results obtained are compared with statistics obtained by structural filtering, NDVI method, and spectral segmentation method. The excellent potential surface water areas can be extracted by using the proposed method.
\end{abstract}

Keywords: colour transformed clustering; LISS III data; normalised difference water index.

Reference to this paper should be made as follows: Parveen, R., Kulkarni, S. and Mytri, V.D. (2019) 'Colour transformed clustering-based water body extraction using IRS-1C LISS III image', Int. J. Spatio-Temporal Data Science, Vol. 1, No. 1, pp.84-97.

Biographical notes: Rubina Parveen is a Research Scholar from the VTU. She completed her BE in ECE (2004) and MTech in Communications (2008). Her main interesting research areas are digital image processing, geo-spatial information science, satellite image analysis and geo-spatial feature extraction. 


\begin{abstract}
Subhash Kulkarni completed his MTech in Electronic Design and Technology from the CEDT IISc Bangalore, in 1995 and $\mathrm{PhD}$ from the Department of E\&ECE, IIT Kharagpur in 2002. He is a Fellow of Institute of Engineers and Fellow of Institute of Electronics and Telecommunication Engineering. His areas of interest are Vedic math, image processing and signal processing.
\end{abstract}

V.D. Mytri completed his MTech in Communication Engineering in 1980 from the Indian Institute of Technology, Madras and PhD in Digital Communication in 1988 from the Indian Institute of Science, Bangalore. He has held various positions in the field of academics: as Dean, Chairman, Board of Studies for Post Graduate Engineering, Chairman, Board of Examinations in Gulbarga University and also VTU Belgaum. He has also held the academically-coveted positions in VTU as Executive Council and Academic Senate member. His area of interest is image processing.

This paper is a revised and expanded version of a paper entitled 'Extraction and delineation of water bodies from IRS-1C LISS III imagery' presented at 13th International IEEE India Conference INDICON 2016, IISc, Bengaluru, 16-18 December 2016.

\title{
1 Introduction
}

Water extraction is significant because water plays a vital role in ecological balance (Parveen et al., 2016) and conservation of water sources (Zahraei et al., 2016) is a major task. Water resources management and continuous monitoring are essential for the reason that water always appears diverse (Qiao et al., 2012) in nature and is present on surface as well underground. Better management of water is necessary as surface water is a scarce resource. Management of water bodies and planning of water distribution requires good and reliable information on water resources (Bastiaanssen et al., 2000). Shadow and water have similar spectral responses. Because of this surface water extraction is not so simple. There are various approaches to extract water from linear imaging self-scanning sensor (LISS) III imagery but every approach has its own merits and demerits. Statistical classification based on features is highly dependent on the image acquisition time and conditions. Results are less accurate with unsupervised classification. For accurate visual interpretation of water areas high-resolution image is required. Coastal vulnerability index and mean shift algorithm is used for sea level rise assessment (Rao et al., 2008; Marfai et al., 2008). A knowledge-driven GIS of weighted linear combination is used for groundwater identification (Manap et al., 2013). Watershed segmentation algorithm based on one-band gradient function is used for hyper spectral images (Tarabalka et al., 2010). Environmental and hydrological factors have to be known for identification of each watershed zone (Donia, 2013). Water extraction algorithm like normalised difference water Index (NDWI) (McFeeters, 1996) is highly subjective. River identification is mostly done by skeletonisation process (Nath and Deb, 2010). Modified normalised difference water index enhances shallow of water regions (Mishra and Prasad, 2015. Many methods available in the literature for water extraction are based on a single model and use either spatial or spectral information.

Spatial and spectral information simultaneously can be used for improved identification of water body. Objective of this paper is to segment the image based on 
colour transformed clustering (Chitade and Katiyar, 2010; Plataniotis and Venetsanopoulos, 2013) method to automatically identify number of homogeneous regions (Askari et al., 2013) and further extracting the surface water bodies from the similar spectral signature areas (Feyisa et al., 2014). This work is an extended work of water extraction based on spectral characteristics (Parveen et al., 2016). Features with similar spectral signatures other than water bodies like shadows (Lu et al., 2011; Arévalo et al., 2008) are erroneously considered as water bodies. So, in order to get improvement in accuracy of water area extraction and reduce the mean square error (MSE) further extensive work has been carried out and is proposed in this article. Objective of the proposed work is identification of homogeneous clusters, extraction of suspected water areas followed by classification into water and non-water areas and finally, delineation of water areas by marking the boundaries to extract pure water pixels. Results are found to be more satisfactory. Proposed work in this paper can be summarised to three groups: clustering and identification of spatially (Richards and Richards, 1999) and spectral homogeneous features of the input LISS III image, verification of water existence in each cluster, extraction of only surface water bodies from that cluster and finally, marking the boundaries for delineation of water areas. Visual interpretation resultant images provide the best delineation of surface water areas of different sizes. Section two gives the details of the dataset used for the study. Section three shows the methodology used for the proposed work, with spatial and spectral segmentation. Section four demonstrates the results and comparison of these results with existing techniques. In this section delineation of water bodies and experimental evaluation is also discussed. Next section of the article shows the details of dataset and software used for the proposed work.

\section{Data details}

Data used for the study belongs to Indian remote sensing satellite resource sat-1C (IRS-1C) satellite LISS III imagery. Image frames belong to Yadgir and Kalaburagi district area, Karnataka state, India. These areas are dry and highly heterogeneous geographically. Vegetation is sparse and agriculture is mostly rain-fed. Different images acquired on 11th April 2006, 20th October 2006 is used for the analysis. LISS III is a multi-spectral data; it has four band bands with 23.5-meter spatial resolution and a swath of $141 \mathrm{~km}$. These images are ortho-rectified and are available in different format. Figure 1 shows the input sub-image for analysis.

LISS III camera provides multi-spectral data in 4 bands which has visible (two bands) and near-infrared (NIR) (one band) and fourth band (shortwave infrared band). The image frame acquired on 11th April 2006, 20th October 2006 has an approximate dimension of 7,067 pixels by 7,055 pixels. Reading the data with enormous size is bit difficult in MATLAB and low resolution of the data makes analysis a bit challenging. For better visualisation and quick analysis, input image is divided into 36 subsets. Each image sub- data is smaller in size approximately $333 * 333$ pixels. Figure 1 shows a significant amount of geo-spatial information. All the analysis is done using MATLAB image processing tool. 
Figure 1 Subset of the image frame acquired on 28 October 2006 (see online version for colours)

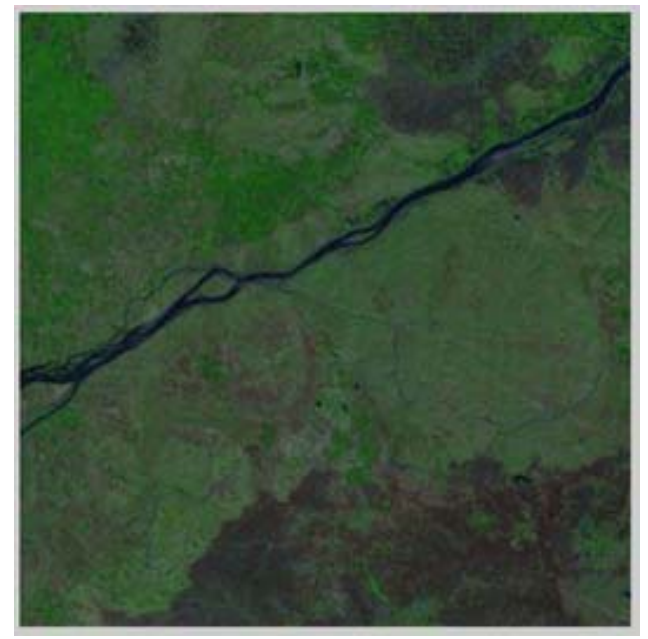

\section{Methodology}

The delineation of water boundaries precisely depends on the resolution of data (Mishra and Prasad, 2015). Resolution of LISS III data less makes the analysis task perplexing. Segmenting LISS III data for extraction of smaller water bodies with more accuracy is challenging. Reflective differences of all the four bands are used to differentiate surface water area, spectral signatures and other features. Presence of mixed pixels makes the reflection of water different in different areas. Mixed pixels may contain impurities like the shadow which makes the task difficult. To minimise the effect of this problem, study image is subjected to colour transformation (Dey et al., 2010) clustering initially. Six clusters are made to group the consistent features. Similar reflectance and spatially homogeneous regions are separated. Every cluster is further subjected to spectral segmentation (Parveen et al., 2016). This twin segmentation helps to separate pure water pixels from non-water pixels. The Methodology of the proposed work (as in Figure 2) shows steps involved in analysis, sequentially.

\subsection{Spatial segmentation}

The Goal of the spatial segmentation is to segment homogeneous geo-spatial features available in the image broadly. The brightness of input image pixels visually depicts the presence of water bodies, vegetation and land mass. The RGB image is converted into L $* a * b *$ colour space to distinguish colours easily. This colour transformation (Borsotti et al., 1998) enables the user to quantify colour differences visually. The $L^{*} a * b *$ colour space consists of a luminosity layer ' $\mathrm{L}^{*}$ ', chromaticity layer ' $\mathrm{a}$ ', and chromaticity layer ' $b^{*}$ '. Colour information of the image is in the ' $a *$ ' and ' $b^{*}$ ' layers. ' $\mathrm{L}^{*}$ ' and ' $\mathrm{a}^{*}$ ' layers represent where the colour falls along the red-green axis. ' $b$ ', layer represents where the colour falls along the blue-yellow axis. Difference between the colours is measured using Euclidean distance metric. Segmentation of the colours in $0 \mathrm{a}^{*} \mathrm{~b}^{*} 0$ 
Space is done by separate clustering group of objects (Blaschke et al., 2004). If the input image exhibits any spectral difference that results in noticeable colour distortion. A heuristic K-means algorithm (Singh et al., 2013) clustering is employed for cluster formation. Let $\mathrm{I}$ be the input image matrix with $\mathrm{m} * \mathrm{n}$ size and can be grouped into $\mathrm{k}$ number of clusters. A is the matrix with $n * k$ with 0 and 1 entry values. In I matrix a (i,j) $=1$ if and only if column $\mathrm{i}$ of $\mathrm{I}$ is assigned to group $\mathrm{j}$ of $\mathrm{k}$ groups. The columns of $\mathrm{m} * \mathrm{k}$ matrix are the centroids calculated by $\mathrm{C}$ using equation (1).

$$
\mathrm{C}=\mathrm{IA} \text { diagonal (1'nA) }-1
$$

$\mathrm{D}=\mathrm{I}-\mathrm{CA}^{\prime}, \mathrm{D}$ is the distance between columns of I and associated centroids CA. The Euclidean distance metric is used. Each object has a location in spatial domain and partitions are done by the k-means algorithm. Six numbers of clusters are specified for portioning. A distance metric is generated to quantify the closeness of two objects. Objects are pixels with ' $a$ ' ' and ' $b$ '' layer values. Objects in the study image are clustered into six clusters using Euclidean distance metric. Each centroid is the mean of the points in that cluster. K-means returns six indexes corresponding to six clusters. Cluster index is used to label every pixel in the image. Pixel labels are used to separate objects by colour. Six resultant images are generated. By visual observation, every image in Figure 3 depicts various geographical features like dense vegetation, rocky land and water bodies, small trees, rain-fed vegetation, irrigated vegetation and open area.

Figure 2 Flow diagram of the proposed methodology (see online version for colours)

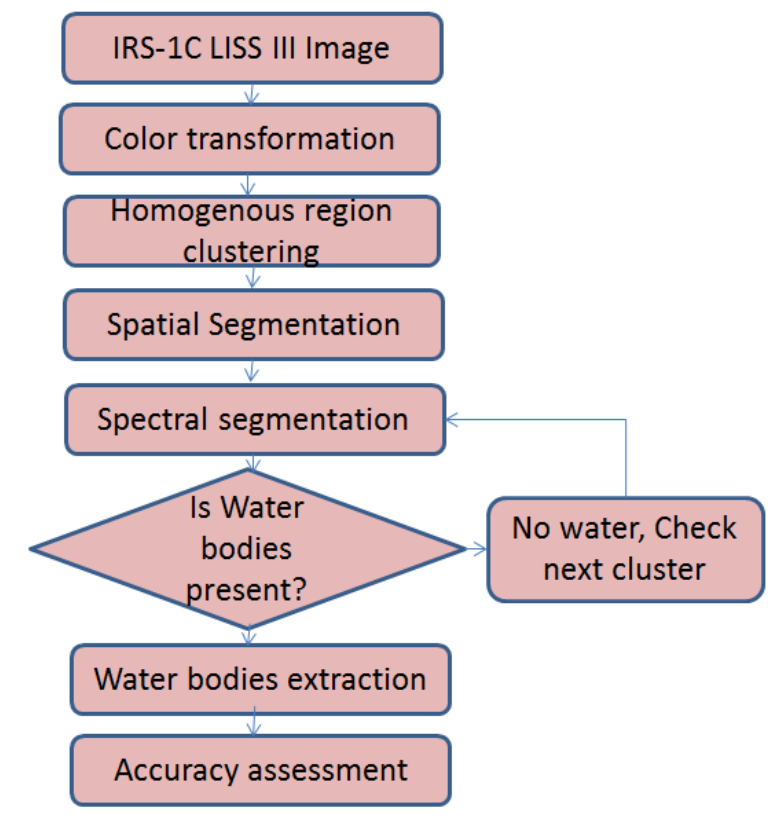


Figure 3 Six segmented clusters, (a) cluster 1 (b) cluster 2 (c) cluster 3 (d) cluster 4 (e) cluster 5 (f) cluster 6 (see online version for colours)

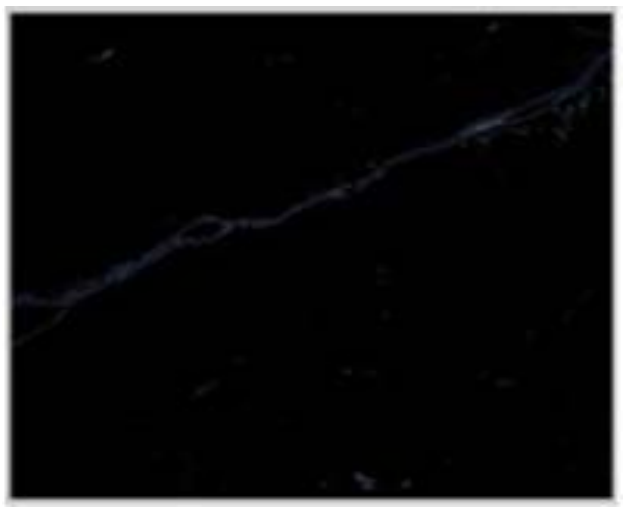

(a)

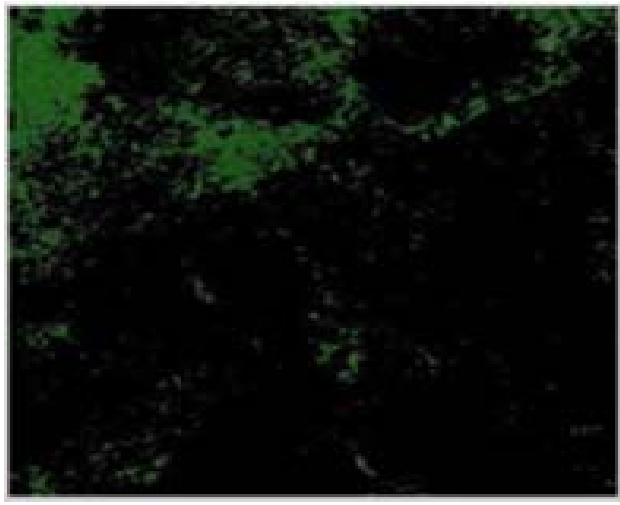

(c)

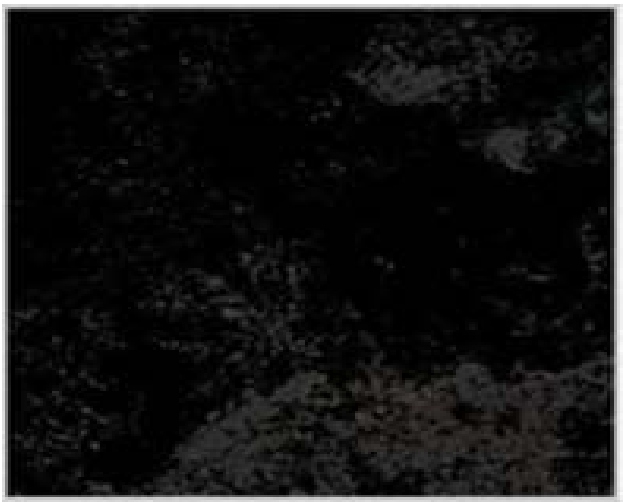

(e)

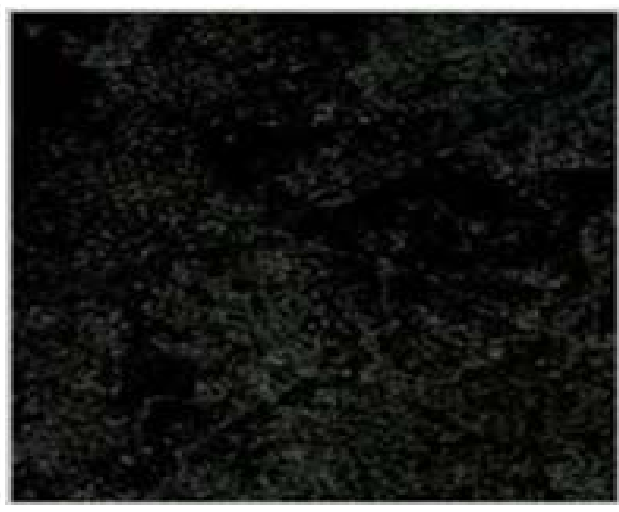

(b)

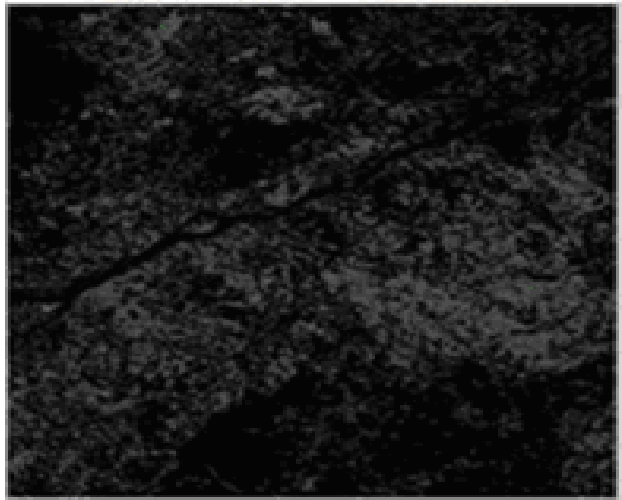

(d)

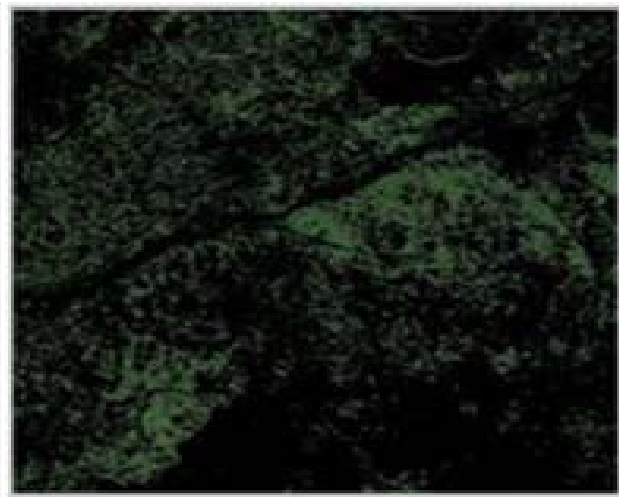

(f)

Note: Each cluster depicts one or more geo-spatial features. 
The percentage of occupancy of homogenous geo-spatial features in the image is tabulated in Table 1. Water bodies are the objects of interest. Contents of these clusters have water and non-water features. Each cluster is checked for the availability of water. According to methodology shown in Figure 2, if the water pixels are present in that particular cluster, then water area is extracted else next cluster is checked for the presence of water pixels in the following section.

Table 1 Features and area of occupancy of each feature in the image

\begin{tabular}{lccc}
\hline Sl no. & Spatial feature & $\begin{array}{c}\text { Number of pixels in the cluster } \\
\text { 1 }\end{array}$ & $\begin{array}{c}\text { The percentage area of } \\
\text { occupancy }\end{array}$ \\
2 & Cluster 1 & 2,673 & 2.410518627 \\
3 & Cluster 2 & 19,268 & 17.37593449 \\
4 & Cluster 3 & 12,776 & 11.52143134 \\
5 & Cluster 5 & 31,424 & 28.33824816 \\
6 & Cluster 6 & 17,511 & 15.79146714 \\
Total & & 27,237 & 24.56240024 \\
\hline
\end{tabular}

\subsection{Spectral segmentation}

In this section, all the clusters are subjected to spectral segmentation (Richards and Richards, 1999). It is visually observed that cluster 1 [Figure 3(a)] shows the presence of water along with the presence of shadow pixels. Features in cluster 1, occupy $2.4 \%$ of the total area. Water pixels can be separated from non-water pixels by brightness values of the pixels in the cluster. Gray threshold is performed (Parveen et al., 2016) by considering spectral properties of the multi-spectral band. Resultant clusters are median filtered for removing the hazy effect. Band indexing is performed and water pixels in the cluster are separated by using the equation (2) for an image $I(i, j)$.

$$
\text { Water index, } R(\mathrm{i}, \mathrm{j})=(4 / \mathrm{pi} * \arctan (\mathrm{BI}))
$$

Where, band index, $\mathrm{BI}=(\mathrm{b}-\mathrm{g}) /(\mathrm{b}+\mathrm{g}) . \mathrm{b}$ is the Blue band and $\mathrm{g}$ is the green band. Inverse tangent function ( $\arctan$ ) is used to return the angle whose tangent is given by the normalised BI. Linear normalisation is done to transform pixel values into the same non-dimensional range $0-1$. Resultant image Figure 4 gives only surface water gray-scale pixels. Figure 4 shows the water areas extracted from cluster 1 .

By visual observation Figure 4 depicts only clear water pixels. The continuous line in the image represents the river. Other clusters don't show the presence of water availability visually, but still, every cluster is checked for water availability for extracting even smaller traces of water. All the remaining clusters are shown in Figures 3(b) to 3(f) are verified individually to extract the water pixels. If water pixel is present in any other clusters also, then that clusters value is also considered for calculation of overall surface water content of the study. Achieved results are analysed and compared with the existing methods available in the literature in the next section. 
Figure 4 Water extracted from spectral segmentation of the cluster $13 \mathrm{a}$

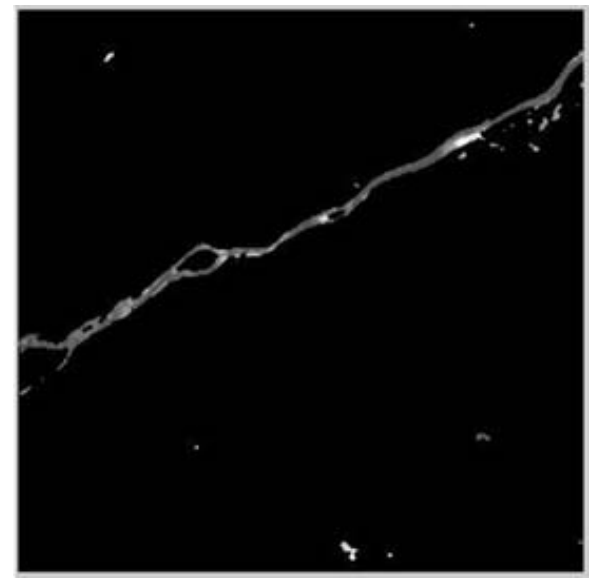

\section{Results and comparison}

Brightness values of the pixels in the cluster are grouped based on the threshold value. In this stage pixels with similar spectral signature, non-water pixels and impure pixels are eliminated. Binarisation of the processed gray image is done, concerning zero, to make a selection of threshold optimal. The standard value of the threshold for water extraction is from 0 to 0.2 for normalised difference band indexing (NDWI) (Kaplan and Avdan, 2017). So, an optimal threshold (T) 0.2 is predefined for water extraction. The decision about water pixels is taken based on a value of the image pixels value crossing the threshold value. All the values above the threshold are changed to 1 and below the threshold to 0 . All the non-water pixel values become 0 and all the water pixel values become 1 by reducing the effects of negative values. Further, these results are compared with standard methods for surface water extraction.

\subsection{Comparison}

Statistical results obtained by the proposed method are compared with three former methods like structural filtering, NDWI (Qiao et al., 2012) and spectral band indexing. These previous methods segment some shallow water areas as background, which is considered as non-water subjectively. In filtering method, structuring filtering element is used for performing morphological operations (Pesaresi and Benediktsson, 2001). Same shape and size parameters are used which are sensitive to required shapes in the processed image. Every pixel in the study image is equated to with its neighbouring pixel. Pixel value adjustment is made depending upon the gamma values. Resultant image of structural filtering method is shown in Figure 5(a). In NDWI method, NDWI reduces the reflectance of the NIR and increases reflectance in the green wavelength, thus increases the reflectance properties of water yielding improved extraction of deep water bodies but the shallow water is poorly detected. Equation (3) is used for water extraction by NDWI method. NDWI processed image is shown in Figure 5(b). 
N DW I = (Green band - N IR band $) /($ Green band + N IR band $)$

Spectral band indexing method (Parveen et al., 2016) calculates the water by using concepts of shadow extraction (Sarabandi et al., 2004; Askar et al., 2002). Appropriate arctan approximation is taken to limit the intensity shift caused by a shadow. Spectral signatures of shadow and water are similar. The resultant image is shown in Figure 5(c). Finally, resultant binary image from the proposed method is demonstrated in Figure 5(d). Clear river water along with its shallow area is observed in Figure 5(d). Output depicts water with sharp shorelines.

A standard threshold value of 0.2 is kept constant, throughout the study. To maintain uniformity in analysis and to maintain the noise and true positive results optimal in the processed images, the same threshold is used for all the technique. The qualitative report shows a distinct line of the river in the processed image by the proposed method in Figure 5(d). Other than that, the water line is observed in spectral band indexing method, but shallow water lines are not clear.

Figure 5 Resultant images obtained by the different segmentation techniques, (a) image obtained by structural filtering (b) image captured by NDWI (c) image obtained by spectral band indexing (d) image captured by the proposed method

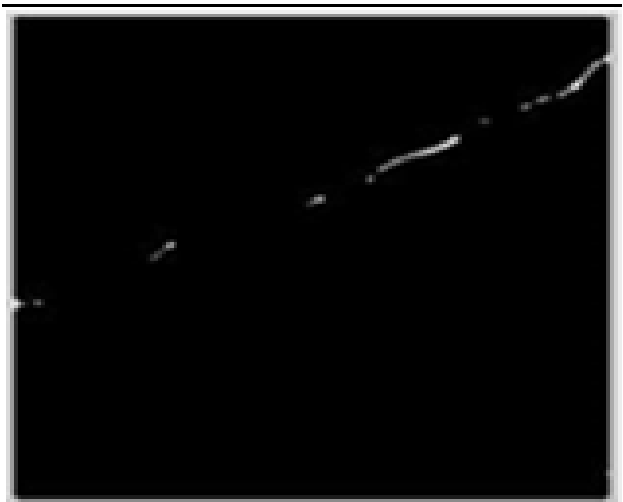

(a)

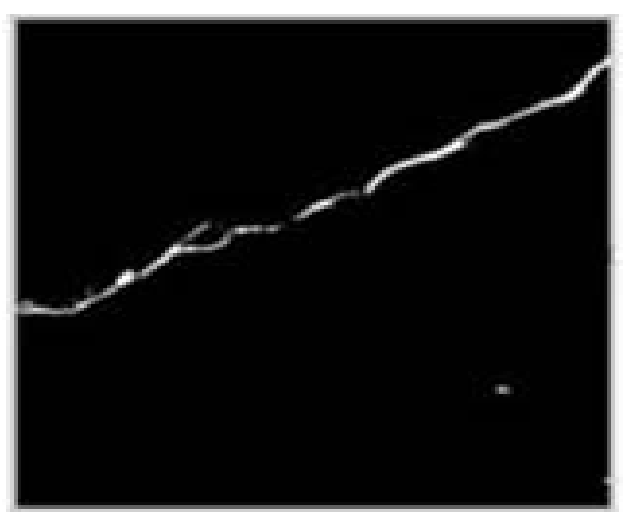

(c)

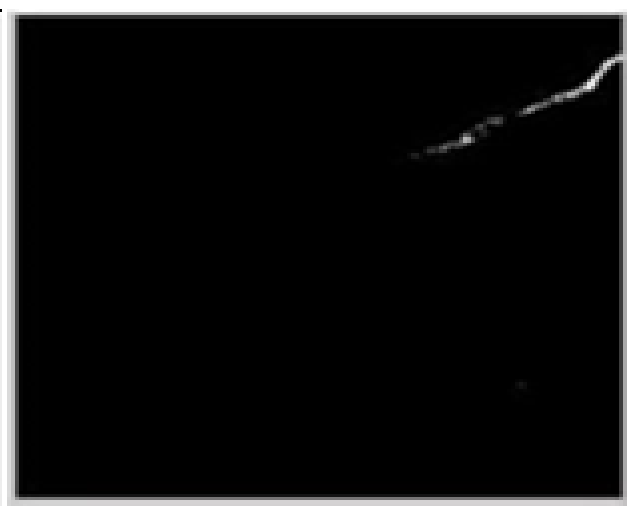

(b)

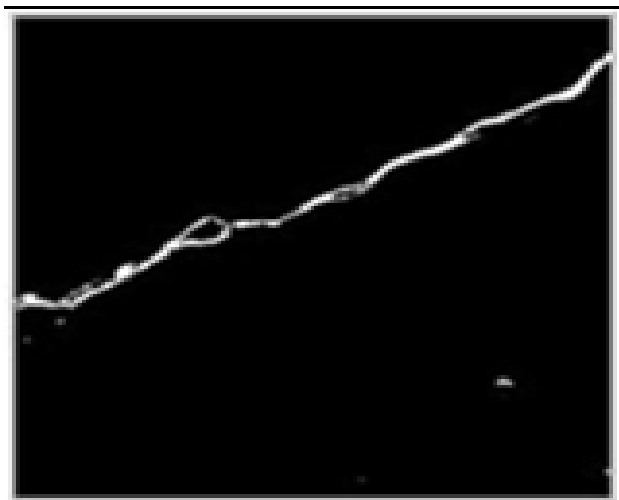

(d) 
The river can be detected with less accuracy visually in the resultant image obtained by structural filtering method in Figure 5(a). The river is not visible by NDWI method in Figure 5(b). Water lines in Figure 5(c) are almost complete but broken. Continuity of the river can be seen only in Figure 5(d) obtained by the proposed method as compared to resultant images obtained by other means.

Quantitative analysis helps to perceive processed image quality automatically. Mathematical deviations between the input image and resultant images provide an objective assessment. So, the number of water pixels, the percentage of water occupancy, entropy, peak signal to noise ratio (PSNR) and MSE are calculated in each case and results are tabulated in Table 2.

Table 2 Quantitative analysis and comparison of the proposed method with other techniques

\begin{tabular}{lccccc}
\hline & & & & \multicolumn{2}{c}{ Technique } \\
\cline { 5 - 6 } Sl no. & Parameter & $\begin{array}{c}\text { Structural } \\
\text { filtering }\end{array}$ & NDWI & $\begin{array}{c}\text { Spectral } \\
\text { band } \\
\text { indexing }\end{array}$ & $\begin{array}{c}\text { Proposed } \\
\text { method }\end{array}$ \\
\hline 1 & Water pixels & 300 & 250 & 1264 & 2673 \\
2 & Percentage water area & 0.2705 & 0.2255 & 1.1399 & 1.5673 \\
3 & Entropy & 0.0550 & 0.0231 & 0.0899 & 0.1164 \\
4 & PSNR & 68.1215 & 24.0994 & 24.0995 & 24.1680 \\
5 & MSE & 0.0101 & 254.998 & 254.9946 & 251.0033 \\
\hline
\end{tabular}

1 The area of water bodies extracted by structural filtering method is $0.27 \%$, NDWI method is $0.22 \%$, spectral band indexing method is $1.13 \%$ (Parveen et al., 2016; Mishra and Prasad, 2015) and proposed method is $1.56 \%$. An additional water area of $0.43 \%$ s is extracted by the proposed plan, as compared to spectral band indexing method.

2 High entropy value shows that resultant image is complex and texturally not uniform. Entropy value is 0.116 ; high entropy value obtained for the proposed method indicates the presence of more correctly detected pixels. Presence of more white pixels depicts that even shallow water areas are extracted.

3 PSNR values are used to access presence of non-water pixels like the shadow or any other darker objects. PSNR value is high and MSE is low in structural filtering; however, water lines are not clearly extracted. PSNR is high (24.1680) and MSE is low (251.0033) in case of the proposed method than compared to NDWI (Xu et al., 2006), spectral filtering method.

4 Therefore, fast-tracking of pure water bodies visually can be done by using the proposed method with higher statistical accuracy.

\subsection{Delineation of water bodies}

Lastly, exact boundaries of water are traced out by performing morphological operations from the image $5 \mathrm{~d}$.

Delineation of water areas is an essential task for quick water analysis. A $5 * 5$ disc structuring element is used for morphological dilation, to cluster homogeneous groups of 
water. The boundaries of water are seen in blue colour in Figure 6. This process helps hydrologist to visualise surface water automatically and accurately.

Figure 6 Surface water is delineated with traced water boundaries obtained by the proposed method (see online version for colours)

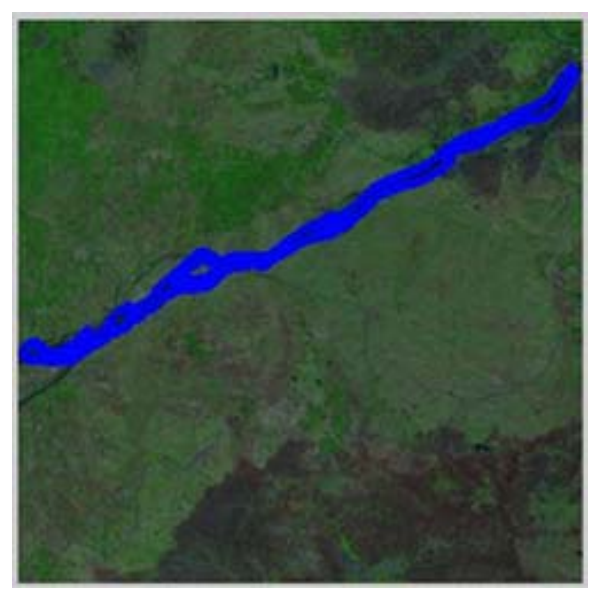

\subsection{Experimental analysis}

The proposed algorithm is tested on the all the sixteen sub-images of two multi-temporal input image frame acquired on 28th October 2006 and 11th April 2006. These multi-temporal image frames are geo-rectified. Results obtained by using sub-image from image frame received on 11th April 2006, is exhibited for the experimental analysis as shown in Figure 7 , this can be used to study the seasonal changes also. Same geographical location is chosen for analysis and to study periodic changes in surface water areas during six months.

Image shown in Figure 7(a) is the input image. Figures 1 and Figure 7(a) are geo rectified LISS III multi-temporal data of the same area. Execution of the proposed algorithm using Figure 7(a), water areas are observed in cluster 1 as shown in Figure 7(b) to Figure 7(e) shows the output of filtering method, NDWI and, spectral band indexing method respectively. Continuity of the river can be seen in Figure 7(e), but less number of pixels is extracted as compared to water pixels in Figure 7(f). Results obtained by the proposed method depict better continuity of the river line. Figure 7 (f) shows $0.78 \%$ of surface water occupancy.

Finally, pure water pixels are obtained by using two geo-rectified multi-temporal images. These pixels depict the changes in surface water availability in study area. Figure 5(d) shows $1.56 \%$ of water occupancy, on 28th October 2006. Figure 7(f) shows $0.78 \%$ of water occupancy, on 11 th April 2006. The 0.78 portion of increased water availability in Figure 1 when compared to Fig. 7a, within six months of the period, due to phenological changes or rainy season. Nearly $0.43 \%$ of additional water areas existing in the study image is detected by the proposed method, as compared to other methods. By implementing this algorithm on images acquired on 28th October 2006 and 11th April 2006, it is observed that availability of surface water is almost doubled from April to 
October in that particular geographical area. These results can be used for change detection also.

Figure 7 Resultant images obtained by using different segmentation techniques for input geo rectified sub image acquired on 11th April 2006, (a) geo-rectified sub-image acquired on 11 April 2006 (b) gray-scale image cluster showing the presence of water, obtained by the proposed method (c) image captured by structural filtering (d) image obtained by NDWI (e) image obtained by spectral band indexing (f) image obtained by proposed method (see online version for colours)

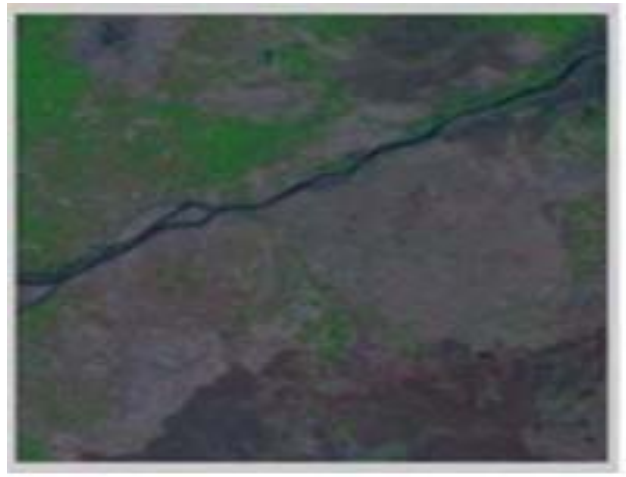

(a)

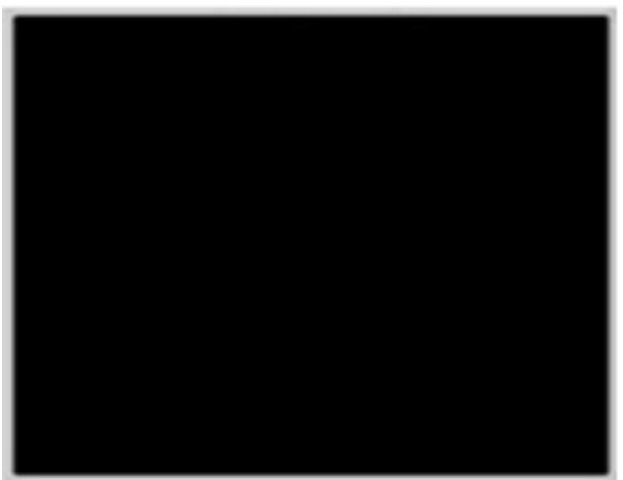

(c)

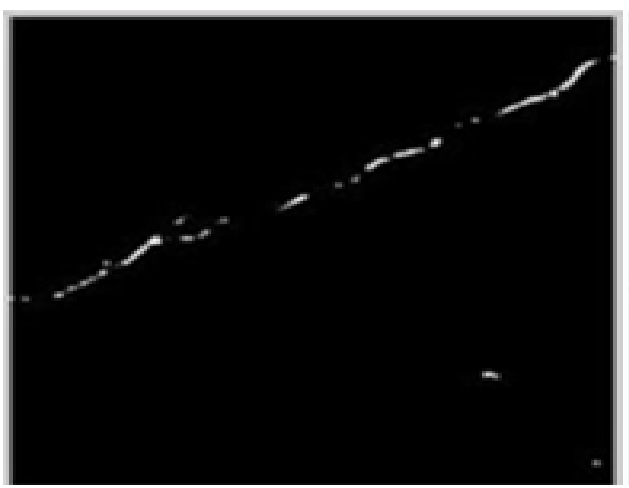

(e)

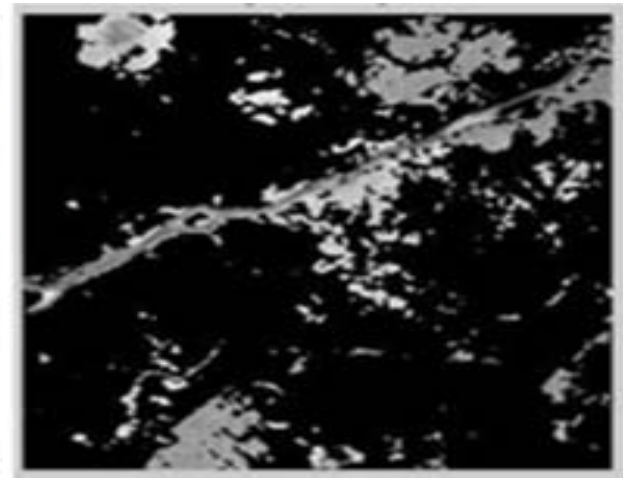

(b)

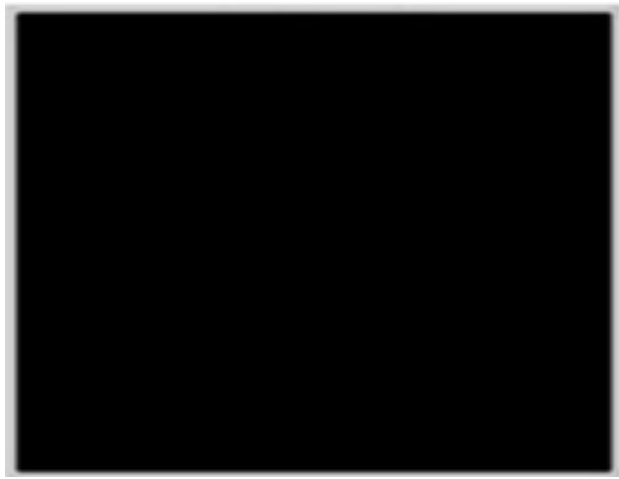

(d)

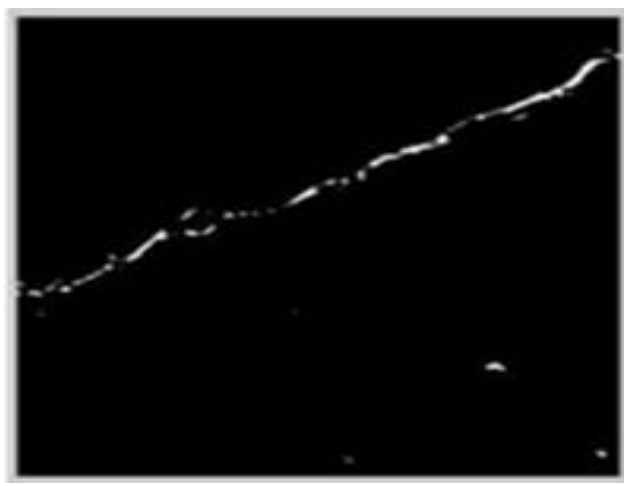

(f) 


\section{Conclusions}

An automatic approach to extract the surface water body from an IRS-1C satellite LISS III imagery is presented in this paper, by using colour transformed spectral segmentation technique. This method is implemented by using a function that merges both spatial and spectral characteristic properties of the geo-spatial features to extract water bodies. This algorithm is successful in demonstrating and evaluating surface water potentiality of any area. Quality assessment by the proposed method is done visually and found to be satisfactory. Statistical results obtained by the proposed method are compared with three former methods like structural filtering, NDWI and spectral band indexing. These former methods segment some shallow water areas as background, which is considered as non-water subjectively. Therefore, qualitative judgment is done by considering actual spectral profiles. Dual segmentation based on spatial colour transformation along with spectral indexing is found to be useful in mapping surface water areas. Proposed algorithm came up as an automatic and an accurate method for finding the surface water, which is very much needed for hydrological applications. Interference of impure pixels is the major setback; this problem can be taken care in the future study.

\section{References}

Arévalo, V., González, J. and Ambrosio, G. (2008) 'Shadow detection in colour high-resolution satellite images', International Journal of Remote Sensing, Vol. 29, No. 7, pp.1945-1963.

Askar, S., Kauff, P., Brandenburg, N. and Schreer, O. (2002) 'Fast adaptive upscaling of low structured images using a hierarchical filling strategy', in Video/Image Processing and Multimedia Communications 4th EURASIP-IEEE Region 8 International Symposium on VIPromCom, IEEE, pp.289-293.

Askari, G., Xu, A., Li, Y. and Alavipanah, S.K. (2013) 'Automatic determination of number of Homogenous regions in SAR images utilizing splitting and merging based on a reversible jump MCMC algorithm', Journal of the Indian Society of Remote Sensing, Vol. 41, No. 3, pp.509-521.

Bastiaanssen, W.G., Molden, D.J. and Makin, I.W. (2000) 'Remote sensing for irrigated agriculture: examples from research and possible applications', Agricultural Water Management, Vol. 46, No. 2, pp.137-155.

Blaschke, T., Burnett, C. and Pekkarinen, A. (2004) 'Image segmentation methods for object-based analysis and classification', in Remote sensing image analysis: Including the spatial domain, pp.211-236, Springer Netherlands.

Borsotti, M., Campadelli, P. and Schettini, R. (1998) 'Quantitative evaluation of color image segmentation results', Pattern Recognition Letters, Vol. 19, No. 8, pp.741-747.

Chitade, A.Z. and Katiyar, S.K. (2010) 'Colour based image segmentation using k-means clustering', International Journal of Engineering Science and Technology, Vol. 2, No. 10, pp.5319-5325.

Dey, V., Zhang, Y. and Zhong, M. (2010) A Review on Image Segmentation Techniques with Remote Sensing Perspective, Vol. 38, No. 7A, pp.31-42.

Donia, N. (2013) 'Application of remotely sensed imagery to watershed analysis a case study of Lake Karoun, Egypt', Arabian Journal of Geosciences, Vol. 6, No. 9, pp.3217-3228.

Feyisa, G.L., Meilby, H., Fensholt, R. and Proud, S.R. (2014) 'Automated water extraction index: a new technique for surface water mapping using landsat imagery', Remote Sensing of Environment, Vol. 140, No. 1, pp.23-35. 
Kaplan, G. and Avdan, U. (2017) 'Object-based water body extraction model using sentinel-2 satellite imagery', European Journal of Remote Sensing, Vol. 50, No. 1, pp.143-150.

Lu, S., Wu, B., Yan, N. and Wang, H. (2011) 'Water body mapping method with HJ-1A/B satellite imagery', International Journal of Applied Earth Observation and Geoinformation, Vol. 13, No. 3, pp.428-434.

Manap, M.A., Sulaiman, W.N.A., Ramli, M.F., Pradhan, B. and Surip, N. (2013) 'A knowledgedriven GIS modeling technique for groundwater potential mapping at the Upper Langat Basin, Malaysia', Arabian Journal of Geosciences, Vol. 6, No. 5, pp.1621-1637.

Marfai, M.A., Almohammad, H., Dey, S., Susanto, B. and King, L. (2008) 'Coastal dynamic and shoreline mapping: multi-sources spatial data analysis in Semarang Indonesia', Environmental Monitoring and Assessment, Vol. 142, Nos. 1-3, pp.297-308.

McFeeters, S.K. (1996) 'The use of the normalized difference water index (NDWI) in the delineation of open water features', International Journal of Remote Sensing, Vol. 17, No. 7, pp.1425-1432.

Mishra, K. and Prasad, P. (2015) 'Automatic extraction of water bodies from Landsat imagery using perceptron model', Journal of Computational Environmental Sciences, No. 1, pp.1-9.

Nath, R.K. and Deb, S.K. (2010) 'Water-body area extraction from high resolution satellite imagesan introduction, review and comparison', International Journal of Image Processing (IJIP), Vol. 3, No. 6, pp.353-372.

Parveen, R., Kulkarni, S. and Mytri, V.D. (2016) 'Extraction and delineation of water bodies from IRS-1C LISS III imagery', in 2016 IEEE Annual India Conference (INDICON), IEEE, December, pp.1-5.

Pesaresi, M. and Benediktsson, J.A. (2001) 'A new approach for the morphological segmentation of high-resolution satellite imagery', IEEE Transactions on Geoscience and Remote Sensing, Vol. 39, No. 2, pp.309-320.

Plataniotis, K.N. and Venetsanopoulos, A.N. (2013) Color Image Processing and Applications, Springer Science and Business Media, Verlag, Berlin Heidelberg, New York.

Qiao, C., Luo, J., Sheng, Y., Shen, Z., Zhu, Z. and Ming, D. (2012) 'An adaptive water extraction method from remote sensing image based on NDWI', Journal of the Indian Society of Remote Sensing, Vol. 40, No. 3, pp.421-433.

Rao, K.N., Subraelu, P., Rao, T.V., Malini, B.H., Ratheesh, R., Bhattacharya, S. and Rajawat, A.S. (2008) 'Sea-level rise and coastal vulnerability: an assessment of Andhra Pradesh coast, India through remote sensing and GIS', Journal of Coastal Conservation, Vol. 12, No. 4, pp.195-207.

Richards, J.A. and Richards, J.A. (1999) Remote Sensing Digital Image Analysis, Vol. 3), Springer, Berlin.

Sarabandi, P., Yamazaki, F., Matsuoka, M. and Kiremidjian, A. (2004) In Geoscience and Remote Sensing Symposium, IGARSS'04, Proceedings. 2004 IEEE International, IEEE, Vol. 6, pp.3744-3747

Singh, A., Yadav, A. and Rana, A. (2013) 'K-means with three different distance metrics', International Journal of Computer Applications, Vol. 67, No. 10, pp.13-17.

Tarabalka, Y., Chanussot, J. and Benediktsson, J.A. (2010) 'Segmentation and classification of hyperspectral images using watershed transformation', Pattern Recognition, Vol. 43, No. 7, pp.2367-2379.

$\mathrm{Xu}, \mathrm{H}$. (2006) 'Modification of normalised difference water index (NDWI) to enhance open water features in remotely sensed imagery', International Journal of Remote Sensing, Vol. 27, No. 14, pp.3025-3033.

Zahraei, A., Eslamian, S. and Saadati, S. (2016) 'The effect of water extraction time from the river on the performance of off-stream reservoirs', International Journal of Hydrology Science and Technology, Vol. 6, No. 3, pp.254-265. 